\title{
Millimeter-Scale Liquid Droplet Migration on Solid Surface with Temperature Gradient: A Simulation Investigation
}

\author{
Jingyuan Zheng \\ Oaks Christian High School, Westlake Village, CA, USA \\ Email:1577841371@qq.com
}

How to cite this paper: Zheng, J.Y. (2020) Millimeter-Scale Liquid Droplet Migration on Solid Surface with Temperature Gradient: A Simulation Investigation. Modern Mechanical Engineering, 10, 34-38. https://doi.org/10.4236/mme.2020.103004

Received: December 12, 2019

Accepted: August 7, 2020

Published: August 10, 2020

Copyright $\odot 2020$ by author(s) and Scientific Research Publishing Inc. This work is licensed under the Creative Commons Attribution International License (CC BY 4.0).

http://creativecommons.org/licenses/by/4.0/

\begin{abstract}
In this paper, we established a time-dependent model that investigates the migration behavior of a millimeter-scale liquid droplet on a solid surface with temperature gradient. Both fluid mechanics and heat transfer are incorporated in the model. The Navier-Stokes equation is employed both inside and outside the droplet. Size variation is observed in the transient simulation. Results show that the velocity of the migration is about $1.7 \mathrm{~mm} / \mathrm{s}$ under a temperature gradient of $30 \mathrm{~K} / \mathrm{mm}$. The model is consistent with results with previous literatures.
\end{abstract}

\section{Keywords}

Liquid Droplet, Fluid Mechanics, Navier-Stokes Equation

\section{Introduction}

Millimeter-scale liquid droplet has profound applications in various fields, such as evaporating or electrowetting behavior in bio-medical industry [1] [2], dielectrophoresis or electrochemical reduction in chemistry and chemical engineering [3] [4], combustion characteristics or thermal conductivity in heat transfer [5] [6], scattering or absorptive properties in electromagnetics [7] [8], etc. Specifically, the migration behavior which requires the joint investigation in both heat transfer and fluid flow region is particularly interesting. However, a simplified model that simulates this phenomenon is not present to the knowledge of the authors.

Tseng has done a fundamental study on the movement of various sized micro-liter droplets on a surface subjected to temperature gradients. The histories 
of droplet movement are recorded by high-speed CCD camera and are simulated by numerical methods based on first principle equations. His study indicates that temperature gradients, the change of dynamic receding/advancing contact angles across the droplets, and the flow fields inside the droplet are the key parameters determining the moving behavior of the micro-droplet driven by Marangoni and capillary effects.

Based on Tseng's work, we established a simplified model to investigate the migration behavior of a millimeter-scale liquid droplet on a solid surface, where the underlying surface is assumed to have a temperature gradient along which the droplet migrates. Finite element simulation is applied to our work.

\section{Theoretical Background}

The description of the fluid flow is based on the Navier-Stokes equations, which in their most general form read as the following [9] [10].

$$
\begin{gathered}
\frac{\partial \rho}{\partial t}+\nabla \cdot(\rho \boldsymbol{u})=0 \\
\rho \frac{\partial \boldsymbol{u}}{\partial t}+\rho(\boldsymbol{u} \cdot \nabla) \boldsymbol{u}=\nabla \cdot(-p \boldsymbol{I}+\tau)+\boldsymbol{F} \\
\rho C_{p}\left[\frac{\partial T}{\partial t}+(\boldsymbol{u} \cdot \nabla) T\right]=-(\nabla \cdot \boldsymbol{q})+\tau: \boldsymbol{S}-\left.\frac{T}{\rho} \frac{\partial \rho}{\partial T}\right|_{p}\left[\frac{\partial p}{\partial t}+(\boldsymbol{u} \cdot \nabla) p\right]+Q
\end{gathered}
$$

The first is the continuity equation and represents conservation of mass. The second is the vector equation which represents conservation of momentum. The third describes the conservation of energy, formulated in terms of temperature. This is an intuitive formulation that facilitates boundary condition specifications. $\rho$ is the density (SI unit: $\mathrm{kg} / \mathrm{m}^{3}$ ), $p$ is pressure (SI unit: $\mathrm{Pa}$ ), $\boldsymbol{u}$ is the velocity vector (SI unit: $\mathrm{m} / \mathrm{s}$ ), $\tau$ is the viscous stress tensor (SI unit: $\mathrm{Pa}$ ), $\boldsymbol{F}$ is the volume force vector (SI unit: $\mathrm{N} / \mathrm{m}^{3}$ ), $C_{p}$ is the specific heat capacity at constant pressure (SI unit: $\mathrm{J} /(\mathrm{kg} \cdot \mathrm{K})$ ), $T$ is the absolute temperature (SI unit: $\mathrm{K}$ ), $\boldsymbol{q}$ is the heat flux vector (SI unit: $\mathrm{W} / \mathrm{m}^{2}$ ), $Q$ contains the heat sources (SI unit: $\mathrm{W} / \mathrm{m}^{3}$ ).

$$
\begin{gathered}
\tau=2 \mu \boldsymbol{S}-\frac{2}{3} \mu(\nabla \cdot \boldsymbol{u}) \boldsymbol{I} \\
\boldsymbol{S}=\frac{1}{2}[\nabla \boldsymbol{u}+(\nabla \boldsymbol{u}) T]
\end{gathered}
$$

$\tau$ is the viscous stress tensor (SI unit: $\mathrm{Pa}$ ). $S$ is the strain-rate tensor. The dynamic viscosity, $\mu$ (SI unit: Pa.s), for a Newtonian fluid is allowed to depend on the thermodynamic state but not on the velocity field. All gases and many liquids can be considered Newtonian.

\section{Model Definition}

A schematic illustration of the millimeter-scale liquid droplet migration on solid surface problem under study is shown in Figure 1. The initial radius of the hemisphere is $0.5 \mathrm{~mm}$. The temperate of the upper boundary of the simulation re- 
gion is set to be the ambient temperature. The temperature of the lower boundary of the simulation region is set to change in a linear way with expression $(493-30 * x[1 / \mathrm{mm}])[\mathrm{K}]$.

\section{Results and Discussions}

Figure 2 shows the pressure distribution of the entire simulation domain. The region of the liquid remains at $32 \sim 38$ Pa during the whole simulation time. To confirm the moving of the droplet, we also plot the volume ration of liquid in Figure 3 and it shows that there is no volume change. Since there is no absolute boundary between liquid and air, we define the region where the volume ration is greater than 0.3 to be liquid. The results in Figure 2 clearly show that the millimeter-scale liquid sphere is migrating with a speed of about $1.7 \mathrm{~mm}$ per second. To ensure the accuracy of the finite element simulation, we set the maximum element size to be $0.04 \mathrm{~mm}$ during mesh process.

In order to give more details of the velocity field, the streamline distribution indicating instantaneously tangent to the velocity vector of the flow is presented in Figure 4. The largest curvature occurs at the edge of the liquid. The temperature field also changes with the influence of the liquid migration, with contribution both from the heat transfer from the liquid itself and the ambient air flow (also shown in Figure 4). The simulation results are consistent with previous experimental results in Figure 5 [11].

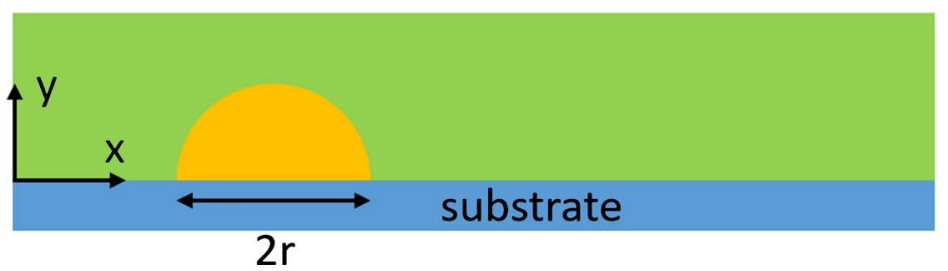

Figure 1. Schematic of the millimeter-scale liquid droplet migration on solid surface problem under study.

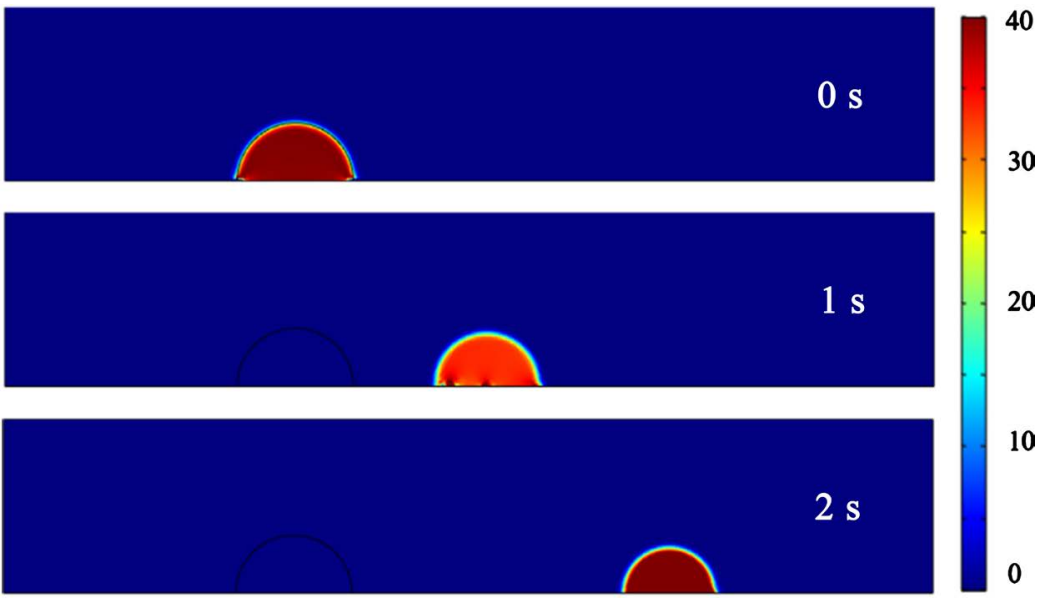

Figure 2. Pressure distribution of the entire simulation region. 


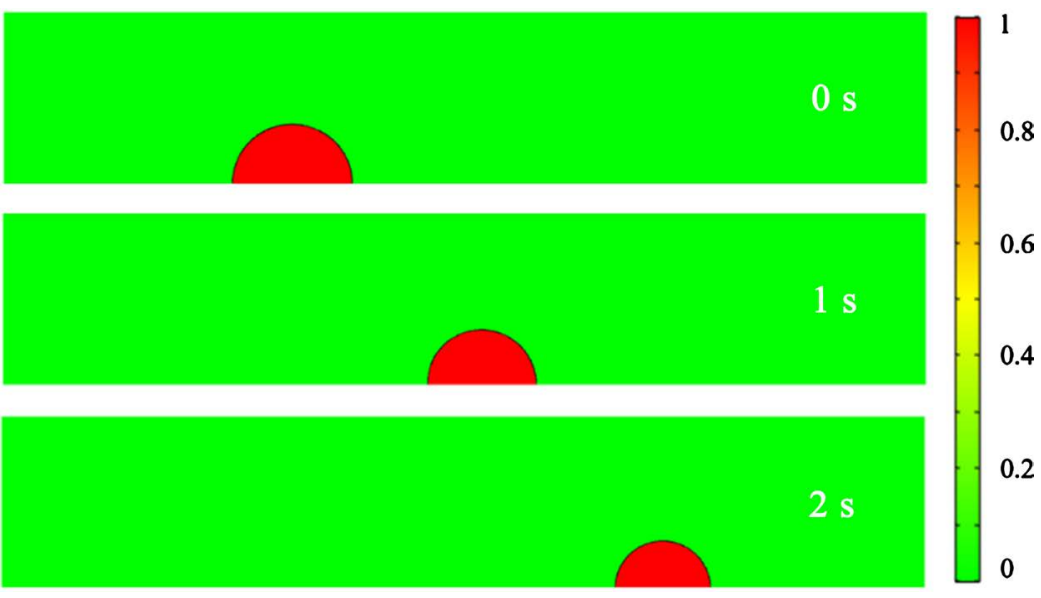

Figure 3. Volume of liquid (larger than 0.3) at different times.
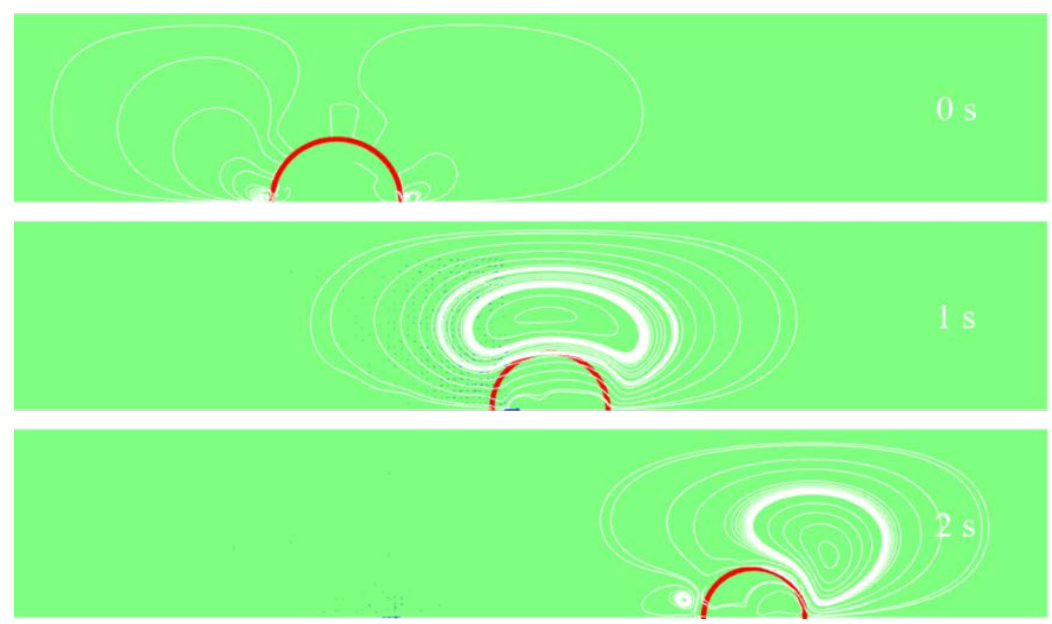

Figure 4. Streamline distribution plot.
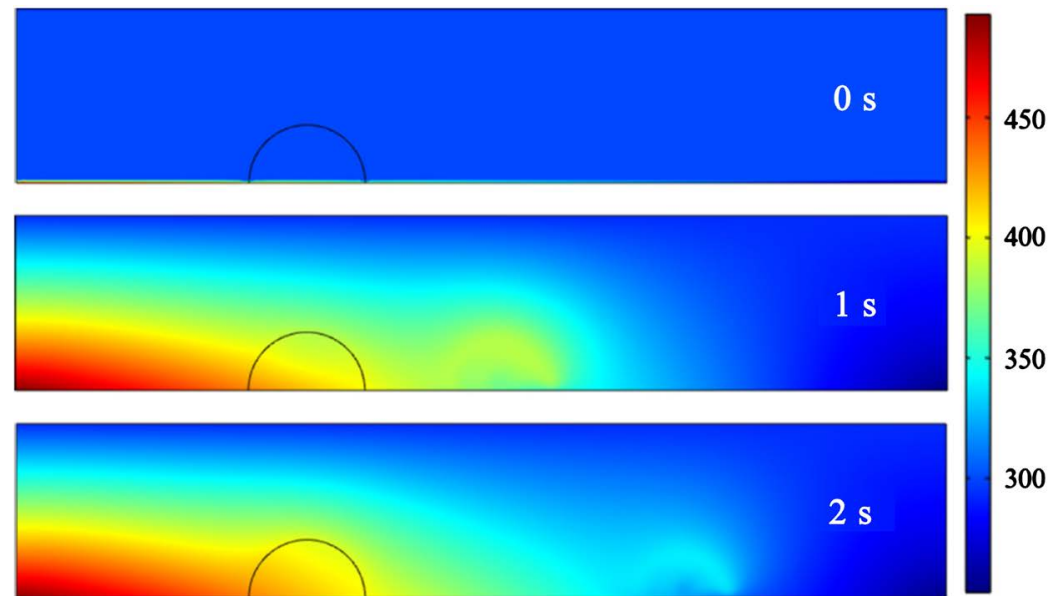

Figure 5. Temperature field distribution of the entire simulation region.

\section{Conclusion}

In conclusion, we firstly build a model that simulates the millimeter-scale droplet migration both in heat transfer and fluid mechanics domain with finite ele- 
ment simulation. Results show that the velocity of the migration is about 1.7 $\mathrm{mm} / \mathrm{s}$ under a temperature gradient of $30 \mathrm{~K} / \mathrm{mm}$ and the largest curvature occurs at the edge of the liquid. The principle can be referred to Tseng's work. We hope that our model finds a way for studying millimeter-scale droplet migration phenomenon under thermal gradient activation. Future work can be done to implement the model in three dimensions that require much more computing power and memory.

\section{Conflicts of Interest}

The author declares no conflicts of interest regarding the publication of this paper.

\section{References}

[1] Sefiane, K. (2010) On the Formation of Regular Patterns from Drying Droplets and Their Potential Use for Bio-Medical Applications. Journal of Bionic Engineering, 7, S82-S93. https://doi.org/10.1016/S1672-6529(09)60221-3

[2] Vergauwe, N., Witters, D., Atalay, Y.T., Verbruggen, B., Vermeir, S., Ceyssens, F., Puers, R. and Lammertyn, J. (2011) Controlling Droplet Size Variability of a Digital Lab-On-A-Chip for Improved Bio-Assay Performance. Microfluidics and Nanofluidics, 11, 25-34. https://doi.org/10.1007/s10404-011-0769-6

[3] Schwartz, J.A., Vykoukal, J.V. and Gascoyne P.R.C. (2004) Droplet-Based Chemistry on a Programmable Micro-Chip. Lab on a Chip, 4, 11-17. https://doi.org/10.1039/b310285h

[4] Kim, B., Kim, J. and Bard, A.J. (2015) Electrochemistry of a Single Attoliter Emulsion Droplet in Collisions. Journal of the American Chemical Society, 137, 2343-2349. https://doi.org/10.1021/ja512065n

[5] Farouk, T.I., Liu, Y.C., Savas, A.J., Avedisian, C.T. and Dryer, F.L. (2013) Sub-Millimeter Sized Methyl Butanoate Droplet Combustion: Microgravity Experiments and Detailed Numerical Modeling. Proceedings of the Combustion Institute, 34, 1609-1616. https://doi.org/10.1016/j.proci.2012.07.074

[6] Yang, T., Kwon, B., Weisensee, P.B., Jin, G.K., Li, X., Braun, P., Miljkovic, N. and King, W.P. (2018) Millimeter-Scale Liquid Metal Droplet Thermal Switch. Applied Physics Letters, 112, Article number: 063505. https://doi.org/10.1063/1.5013623

[7] Yoo, Y.J., Ju, S., Park, S.Y., Kim, Y.J., Bong, J., Lim, T., Kim, K.W., Rhee, J.Y. and Lee., Y.P. (2015) Metamaterial Absorber for Electromagnetic Waves in Periodic Water Droplets. Scientific Reports, 5, Article number: 14018. https://doi.org/10.1038/srep14018

[8] Malouin Jr., B.A., Vogel, M.J. and Hirsa, A.H. (2010) Electromagnetic Control of Coupled Droplets. Applied Physics Letters, 96, Article number: 214104. https://doi.org/10.1063/1.3428787

[9] White, F.M. (2015) Fluid Mechanics.

[10] Schetz, J.A. and Fuhs, A.E. (1999) Fundamentals of Fluid Mechanics. John Wiley \& Sons, New York.

[11] Tseng, Y., Tseng, F., Chen, Y. and Chieng, C. (2004) Fundamental Studies on Micro-Droplet Movement by Marangoni and Capillary Effects. Sensors and Actuators A: Physical, 114, 292-301. https://doi.org/10.1016/j.sna.2003.12.007 